Meta

Journal des traducteurs

Translators' Journal

\title{
Le statut du syntagme dans les dictionnaires généraux monolingues
}

\section{Jean-Claude Boulanger}

Volume 34, numéro 3, septembre 1989

1. Actes du Colloque Les terminologies spécialisées: Approches quantitative et logico-sémantique et 2 . Actes du Colloque Terminologie et Industries de la langue

URI : https://id.erudit.org/iderudit/002109ar

DOI : https://doi.org/10.7202/002109ar

Aller au sommaire du numéro

Éditeur(s)

Les Presses de l'Université de Montréal

ISSN

0026-0452 (imprimé)

1492-1421 (numérique)

Découvrir la revue

Citer cet article

Boulanger, J.-C. (1989). Le statut du syntagme dans les dictionnaires généraux monolingues. Meta, 34(3), 360-369. https://doi.org/10.7202/002109ar d'utilisation que vous pouvez consulter en ligne. 


\section{LE STATUT DU SYNTAGME, DANS LES DICTIONNAIRES GENERAUX MONOLINGUES}

JEAN-ClAUdE BOULANGER

Université Laval, Québec, Canada

1. INTRODUCTION

En linguistique, le terme syntagme est polysémique - homonymique diraient les terminologues - ce qui, à première vue, peut paraittre gênant lorsque des spécialistes des questions de langue discutent entre eux et qu'ils sont d'obédiences différentes. Au moins trois sens peuvent le caractériser. Délaissant le syntagme saussurien et le syntagme chomskyen, je focaliserai mon attention sur le syntagme terminologique, qui, il ne faut pas se le cacher, doit beaucoup à ses devanciers et dont il n'est en somme qu'une spécialisation par restriction de sens comme l'indique le déterminant terminologique qu'on lui accole fréquemment. Le ST sera défini comme étant un groupe de mots séparés par des blancs, syntaxiquement liés et identifiant une notion unique dans un domaine déterminé du savoir. Le syntagme est donc un terme. Il s'oppose au terme simple constitué d'un seul mot graphique (ex. maculature, infographie ou de deux mots ou plus reliés par un trait d'union (ex. page-écran, gros-porteur; arrière-petits-enfants, porte-à-faux) ou une autre marque graphique (ex. : presqu' ile, pop'art, avion + auto ; filtreloccillateur, roll-in/rollout). La notion de «syntagme terminologique», ou plus commodément de «syntagme», est rendue en français par une prolifération de synonymes dont une trentaine ont été catalogués jusqu'à présent (voir Kocourek, 1982: 117). En ce moment, le terme syntagme semble le choix des Québécois tandis que les Français lui préfèrent souvent la forme composée, ce qui n'est pas sans causer quelques difficultés de compréhension entre les chercheurs des deux espaces géographiques. Il faut cependant préciser que dans le milieu des praticiens de la terminologie, c'est le terme syntagme qui l'emporte, que ce soit en Amérique du Nord ou en Europe. Il se trouve aussi dans des écrits lexicographiques et métalexicographiques. Josette Rey-Debove (1971: 112) dénomme syntagme «les entrées formées de plusieurs mots graphiques (simples ou composés) non reliés». Quant à Alain Rey (1988: XIII), il distingue bien une série d'unités complexes parmi lesquelles il reconnaît les syntagmes courants ou terminologiques, les locutions et les proverbes.

Les nuances terminologiques étant évoquées, il reste à rappeler quelques principes terminologiques importants.

1.1 En terminologie, le terme s'oppose au mot. Le premier identifie spécifiquement les unités lexicales des langues de spécialité (LSP) tandis que le second circonscrit les unités lexicales de la langue générale (LG) ou courante. Du point de vue théorique, cette distinction est utile et fonctionnelle.

1.2 Le syntagme terminologique peut être simple, c'est-à-dire correspondre à une structure de base binaire telle que définie par Louis Guilbert (1970: 117-119): ex. banc de neige, permis de conduire, pluies acides, laveur-récupérateur de liqueur, conducteur d'excavateur-compresseur; il peut aussi être complexe, c'est-à-dire dériver d'une structure modèle élémentaire par l'intermédiaire d'une expansion déterminative: ex. papier 
LE STATUT DU SYNTAGME DANS LES DICTIONNAIRS GÉNÉRAUX MONOLINGUES

couché à haut brillant, station mobile terrestre, stabilisation par gradient de gravité, carton gris doublé blanc une face.

1.3 Incontestablement, si l'on se place du point de vue lexical, le syntagme constitue la caractéristique dominante en terminologie. Diverses évaluations fixent à environ $80 \%$ la proportion moyenne des unités synaptiques dans les LSP. Une vérification menée dans deux dictionnaires terminologiques, l'un consacré au vocabulaire des papiers des cartons (Côte: 1983) et l'autre aux appelations d'emplois dans l'industrie papetière québécoise (Côte: 1982), fournit les données suivantes:

- Dans le premier vocabulaire, il y a 610 unités réparties comme suit : 564 syntagmes, 25 termes composés et 21 termes simples. La proportion des unités complexes par rapport aux autres atteint $92,5 \%$.

- Dans le second dictionnaire, 304 termes sont recensés selon l'ordre suivant : 223 syntagmes, 55 termes simples et 26 termes composés. Les ST donc pour $73.3 \%$ de l'ensemble des entrées.1

Si l'on réunit les deux nomenclatures, la moyenne se situe à $82,9 \%$, ce qui confirme d'une manière concrète les projections faites à partir d'autres sources (voir les tableaux 1 et 2).

Tableau 1: Catégories d'entrées dans un DT : nombre

\begin{tabular}{|l|c|c|c|}
\hline DICTIONNAIRES & VPC & AEIPQ & TOTAL \\
\hline TYPES D'ENTRÉES & 564 & 223 & 787 \\
\hline TERMES COMPOSÉS & 25 & 26 & 51 \\
\hline TERMES SIMPLES & 21 & 55 & 76 \\
\hline TOTAL & 610 & 304 & 914 \\
\hline
\end{tabular}


Tableau 2: Catégories d'entrées dans un DT : pourcentage

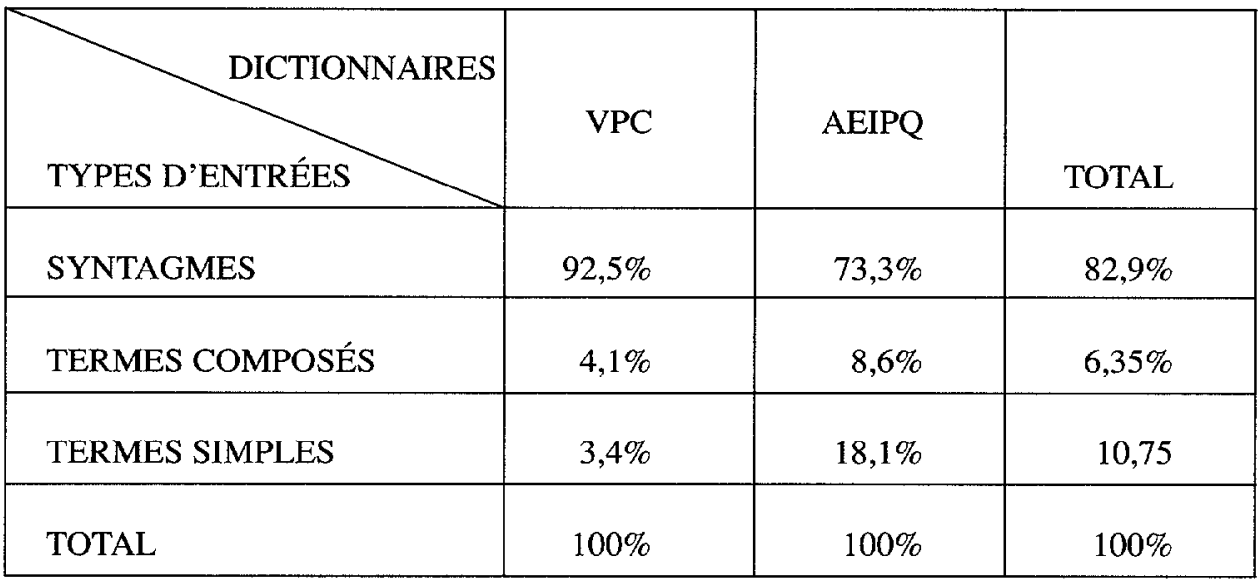

1.4 Le dictionnaire terminologique (DT) est élaboré en s'inspirant de principes onomasiologiques (primauté du concept sur le signe) tandis que le dictionnaire général monolingue (DGM) s'appuie sur une démarche sémasiologique (primauté du signe sur le signifié). Les unités lexicales rassemblées et définies dans les répertoires de langue, y compris les lexies complexes, sont considérées sous l'angle le plus général du lexique: le mot graphique, «alors que dans les DT, les termes sont au moins en théorie - le produit d'une opération onomasiologique de désignation» (Rey, 1987:8). La vision du lexicographe l'amène alors à percevoir le syntagme comme le résultat de la «formation d'une nouvelle unité lexicale complexe par association d'unités lexicales simples empruntées au fonds commun du lexique» (Phal, 1969: 76). De ce fait, il hésitera à faire du syntagme une entrée autonome dans son dictionnaire sauf si celui-ci «est à la fois détaché de sa source et par lui-même important (chemin de fer, repris de justice)» (Rey, 1988: XIII). II est alors traité en véritable mot et logé à sa place alphabétique. Dans tous les autres cas, infiniment plus nombreux, le dictionnairiste refragmentera la synapsie en ses éléments constituants afin de sélectionner un mot-pivot et de reconstruire le syntagme dans la microstructure déployée sous ce mot principal. Pour le terminographe, les séquences syntagmatiques «ont la même cohérence interne et le même caractère de nécessité que chemin de fer. C'est dire que dans le domaine des sciences et des techniques, les cadres de la lexicalisation sont considérablement plus extensibles que ceux du dictionnaire le plus accueillant» (Phal, 1969: 76).

C'est en ayant ces considérations à l'esprit que l'on pénétrera au cœur d'une étude qui croise la trame lexicographique (le contenant) avec la chaîne terminologique (le contenu).

\section{TERMINOLOGIE ET DICTIONNAIRE DE LANGUES}

C'est un fait facilement observable que les DGM ne restreignent pas leur nomenclature aux mots et aux sens d'usage courant. De tous temps, des vocabulaires spécialisés se sont banalisés, vulgarisés; ils sont sortis du cercle de l'usage réservé aux spécialistes pour s'introduire dans les dictionnaires destinés à un vaste public. Il n'est plus guère possible d'ignorer le passage de plus en plus fréquent des terminologies dans la sphère d'influencer des DGM comme en font foi la plupart des préfaces, des introductions ou 
LE STATUT DU SYNTAGME DANS LES DICTIONNAIR/S GÉNÉRAUX MONOLINGUES

des présentations des répertoires récents (voir Boulanger, 1986: 63 et suiv.). La langue propose au locuteur des unités lexicales complexes qu'il est nécessaire d'apprendre et de maîtriser aussi bien que les mots simples (ex. conte de fées, ordre alphabétique, point de vue).

En outre, la majeure partie des refontes ou des mises à jour des dictionnaires généraux repose sur l'introduction du contingent lexical terminologique, les mots de la langue courante se présentant en nombre plus limité.

Par ailleurs, il est quasi impossible de séparer l'étude des termes de l'étude des mots étant donné la possibilité pour les premiers de se transformer en mots (ex. interface, qui $a$ acquis un sens métaphorique: «deux aspects d'un même problème», voir CRLF) et pour les seconds de revêtir l'habit terminologique (ex. : beauté, charme, couleur, étrangeté qui ont émigré vers la physique nucléaire, sans doute sous l'influence de l'anglais). Rien en lexicologie ne permet de distinguer le signifiant LG du signifiant LSP. Deux formes aussi banales que bruit et blanc peuvent s'associer pour donner bruit blanc dont le sens est: «Bruit à spectre continu dont la densité spectrale d'énergie est pratiquement constante dans un intervalle de fréquences spécifié» (Vocabulaire des sciences et techniques spatiales, 1978: 27). Ce syntagme du domaine de l'électromagnétisme possède une charge sémantique très forte qui est loin d'être détectable à partir de l'assemblage fortuit de deux signifiants au sémantisme par ailleurs transparent et polyvalent.

Il est donc naturel de rencontrer des termes dans les DGM. Ils y ont le statut d'entrées indépendantes et cela depuis la naissance de la lexicographie française. En général, ils sont repérables grâce à une étiquette de niveau de spécialisation socioprofessionnelle qui les accompagne. Lorsqu'elle est présente, cette étiquette est coincée quelque part entre l'indicatif du sens (numéro, signe diacritique ou minifigure: losange, carré, cercle) et la définition (ex. chélate, chim., chélicère, zool., chélidoine, bot., chéloide, méd. (GRLF)). La marque d'appartenance technolectale capture le terme et l'insère dans une typologie classologique (cf. Rey, 1987: 8).

\section{LA PLACE DU ST DANS LES DGM}

Même s'ils les discutent, les lexicographes généralistes reconnaissent tous l'importance des unités complexes dans les dictionnaires. Le phénomène est historique car les dictionnairistes ont toujours pris des libertés avec les mots graphiques. Au XVIIIe siècle, pour prendre un exemple et pour ne pas remonter trop loin dans le temps, des syntagmes ont déjà accès au statut d'entrée (ex. bouton de mer, Trévoux, 1771).

Tout en n'ignorant pas l'importance qualitative et quantitative des ST, «le dictionnaire de langue doit effectuer une sélection sévère, sous peine $d$ 'assommer son lecteur de mots et d'expressions (ou syntagmes) désignant des réalités qui ne sont nommées que dans des activités hyperspécialisées de la connaissance. [...] si l'on tient compte du fait que de nombreux termes ne sont pas des mots simples, mais des «syntagmes» - et ceci est vrai de bien des «mots» courants: grand ensemble est traité à part -, les nomenclatures deviennent plus impressionnantes» (Rey, 1985: XXIV). Selon l'estimation du lexicographe robertien, un DGM de 80000 entrées contiendrait environ 500000 syntagmes terminologiques disséminés au travers les articles, une moyenne assez impressionnante par article (cf. Rey, 1985 : XXIV n. 6)

\subsection{LA SÉLECTION DES ENTRÉES}

L'entrée est la principale voie d'accès au dictionnaire de langue. Or, il n'est pas besoin de feuilleter longuement un répertoire pour constater que toutes les adresses lexicographiques n'ont pas la même physionomie. Quatre grands groupes sont facilement repérables: les éléments de formation: préfixes, suffixes, etc. (ex. ana-, -ana), les mots 
simples (ex. musique, francophonie), les mots composés, au sens traditionnel du terme (ex. analyste-programmeur, lave-mains), et les syntagmes (ex. bel canto, repris de justice). Les quatre modèles procèdent de l'inframot au supramot. Les modes de classement reposent sur le déroulement de la séquence graphique dans laquelle intervient ou non une rupture sous la forme d'un signe ou d'un blanc typographique.

Afin de vérifier si les constats visuels répondaient à des propositions théoriques ou méthodologiques communes, j'ai examiné les opinions de quelques lexicographes. En 1971 , les frères Dubois (Larousse) soutenaient que «les entrées lexicographiques doivent être des «mots» compris entre deux blancs typographiques au sens le plus strict. [...] Les mots composés dont les éléments composants sont séparés par un trait d'union (coffrefort), ou dont les éléments ne sont pas séparés (gentilhomme) sont des mots et constituent des entrées. En revanche, les termes composés (syntagmes) dont les composantes ne sont pas réunies par le trait d'union et qui sont ainsi séparées par un blanc ne sont pas reconnues comme des entrées: ainsi pomme de tẹrre, chou rave (contrairement à chou-fleur), compte rendu (contrairement à compte-gouttes), etc. [...] C'est la présence ou l'absence du trait d'union qui définit les mots composés qui peuvent être des entrées et ceux qui ne sont que des sous-entrées (ainsi pomme de terre est à pomme, compte courant est à comp$t e$, etc.)" (Dubois/Dubois, 1971: 62). La forme graphique, basée sur la non-interruption de la séquence de lettres et de signes diacritiques, l'emportait alors sur le sens ou la démotivation étymologique.

À la même époque, pour Josette Rey-Debove (1971: 114), il est clair que suivant le principe de la double structure lexicographique, «qui seul permet de séparer la langue de ses réalisations en discours, tout mot graphique simple ou composé, réduit à sa forme canonique (regroupement des variantes) doit figurer à la nomenclature, et non dans la micro-structure où il ne représente qu'une unité de discours (exemple)». Par ailleurs, tout «syntagme» à sens particulier non déductible de celui de ses composants devrait figurer à la nomenclature (Rey-Debove, 1971: 117).

Les discussions sur la place du syntagme dans le DGM trouvent d'autres échos, notamment chez les métalexicographes. Ainsi, «aucun dictionnaire ne peut se permettre de consacrer une entrée à chacun des ensembles qui pourraient être des mots composés ou des synapsies: en plaçant bateau-citerne, bateau-mouche, etc. à l'intérieur de l'article bateau (malgré les traits d'union), tout comme bateau à moteur et bateau de commerce, etc. le P.R. gagne une place précieuse, qu'il gaspillerait en faisant de chaque composé une entrée» (Collignon/Glatigny, 1978: 67).

Les positions oscillent entre les contraintes créées par la définition du concept de «mot» (point de vue théorique et linguistique) et les raisons proprement matérielles (augmenter le contenu des ouvrages). Aujourd'hui encore, les conventions lexicographiques traditionnelles perdurent dans le choix de la forme lexicale qui servira d'entrée; cette forme est «celle du mot défini par le critère graphique de la séparabilité dans l'énoncé; cela suppose un système de signification idéal selon lequel à l'unicité morphologique du mot correspondrait l'unicité du concept» (Guilbert, 1969:7).

En fait, la sélection des unités qui joueront le rôle de chef d'article repose sur une ensemble de principes aussi divers que le principe commercial et financier (le budget), le principe idéologique (la formation et la personnalité des rédacteurs-lexicographes), le principe du programme dictionnairique (public-cible, taille de la nomenclature) et le principe lexicographique lui-même (rapports entre macrostructure et microstructure lors du traitement de l'article).

Un relevé de la séquence $C H E$ - dans cinq dictionnaires permet d'illustrer concrètement le comportement nomenclatural de la lexicographie française. 
LE STATUT DU SYNTAGME DANS LES DICTIONNAIRS GÉNÉRAUX MONOLINGUES

Tableau 3: Catégories d'entrées dans un DGM : nombre

\begin{tabular}{|l|r|r|r|r|r|}
\hline $\begin{array}{l}\text { DYPES } \\
\text { D'ENTRÉES }\end{array}$ & GRLF 85 & DFP 88 & PR 84 & PLI 88 & PLI89 \\
\hline MOTS SIMPLES & 169 & 129 & 108 & 114 & 122 \\
\hline MOTS COMPOSÉS & 10 & 4 & 10 & 11 & 12 \\
\hline ÉLÉM. DE FORMATION & 9 & 0 & 0 & 0 & 0 \\
\hline SYNTAGMES & 1 & 1 & 2 & 1 & 1 \\
\hline \multicolumn{1}{|c|}{ TOTAL } & 189 & 134 & 120 & 126 & 135 \\
\hline
\end{tabular}

Tableau 4 : Catégories d'entrées dans un DGM : pourcentage

\begin{tabular}{|l|r|r|r|r|c|}
\hline \multicolumn{1}{|l|}{$\begin{array}{l}\text { TYPES } \\
\text { D'ENTRÉES }\end{array}$} & GRLF 85 & DFP 88 & PR 84 & PLI 88 & PLI89 \\
\hline MOTS SIMPLES & $89,4 \%$ & $96,3 \%$ & $90,0 \%$ & $90,5 \%$ & $90,4 \%$ \\
\hline MOTS COMPOSÉS & $5,3 \%$ & $3,0 \%$ & $8,3 \%$ & $8,7 \%$ & $8,9 \%$ \\
\hline ÉLÉM. DE FORMATION & $4,8 \%$ & $0,0 \%$ & $0,0 \%$ & $0,0 \%$ & $0,0 \%$ \\
\hline SYNTAGMES & $0,5 \%$ & $0,7 \%$ & $1,7 \%$ & $0,8 \%$ & $0,7 \%$ \\
\hline \multicolumn{1}{|c|}{ TOTAL } & $100 \%$ & $100 \%$ & $100 \%$ & $100 \%$ & $100 \%$ \\
\hline
\end{tabular}

Les résultats observés n'offrent aucune surprise. Les quatre catégories de vedettes sont représentées dans des proportions que révèlent bien les deux tableaux précédents. La macrostructure est dominée dans une proportion écrasante par les mots simples (unimots). La moyenne atteint $91,2 \%$ (642/704). Les mots composés arrivent en deuxième place, tandis que les syntagmes et les éléments de formation se partagent la troisième ou 
la quatrième position selon les dictionnaires. Le syntagme chemin de fer est présent partout; l'autre syntagme enregistré dans le PR84 est chêne vert. Il n'est pas question de tirer des conclusions défitinives à partir d'un corpus si réduit. Les résultats pourraient varier suivant la tranche nomenclaturale étudiée, ou encore suivant le nombre beaucoup plus grand d'adresses vérifiées ou suivant les dictionnaires dépouillés. Une projection toute intuitive permet de considérer le schéma offert par le GRLF comme étant le plus plausible. En effet, la séquence $C H E$ - ne contient pas beaucoup d'affixes; on y repère plutôt des éléments savants non pris en compte dans les quatre dictionnaires en un volume. Les affixes sont présents en plus grand nombre ailleurs dans ces recueils.

Quant au syntagme, on peut considérer que la diversité de ses formes, son instabilité conceptuelle (dans le cas des syntagmes de discours), sa longueur souvent encombrante, son rôle en langue générale et l'absence de consensus à son égard sont les principaux aspects qu'il faut scruter lorsqu'on le met en rapport avec la lexicographie et les DGM. Si l'on prend, par exemple, le critère de la longueur du syntagme, on remarque que la lexie complexe retenue en entrée ne franchit pas le seuil du schéma de base guilbertien comportant deux, trois ou quatre composants, y compris les joncteurs ou les ligaments syntaxiques. Au delà de cette taille, naine pour qui connaît le ST, les mots complexes ne sont généralement pas admis à figurer comme têtes d'article.

Le lexicographe poursuit avant tout des objectifs synoptiques. Aussi, les longues locutions, les proverbes et les syntagmes sont-ils refoulés le plus souvent dans la microstructure, du moins dans l'état actuel de la lexicographie française. À noter cependant, que les deux premières catégories d'expressions sont toujours accompagnées d'une marque spéciale dans l'article (loc. ou prov.), ce qui n'est pas encore le cas du syntagme figé, non plus que lorsqu'il est répertorié en entrée, ni dans le corps de l'article. Il est sans doute temps d'y voir et de fournir au syntagme son propre fil d'Ariane au sein de l'article de dictionnaire.

\subsection{LA PRÉSENTATION DE L'ENTRÉE SYNTAGMATIQUE}

Une fois que le terme complexe a été sélectionné pour être porté au catalogue macrostructural, il doit être lemmatisé, c'est-à-dire neutralisé. Plusieurs méthodes de présentation du syntagme en entrée ont été répertoriées (voir le tableau 5).

1. Le syntagme se déroule conformément à la succession naturelle de ses éléments constituants (ex. GRLF; big bang, chemin de fer, grand ensemble, Yorkshire pudding). Il donne accès au développement microstructural complet, signe de reconnaissance de l'autonomie du mot d'entrée tant au plan formel que sémantique.

2. La formulation syntagmatique n'obéit pas toujours à l'ordre linéaire discursif naturel. On rencontre, à l'occasion, une vedette complexe dont les éléments ont été permutés. La base est reportée entre parenthèses après l'expansion qui devient la clé d'accès alphabétique. (ex. GRLF : frise (cheval de), Bengale (feu de); PLI : Bénioff (plan de)). La forme d'accès à l'article est aussi bien un nom commun (frise) qu'un nom propre (Bengale, Bénioff), ce qui, dans le dernier cas, est un peu contradictoire dans un dictionnaire de langue. Le développement microstructural complet se déploie ensuite sous l'entrée concrétisant la reconnaissance d'une certaine cohésion entre les composants, mais rejetant l'autonomie complète, du moins en apparence.

3. Le syntagme, offert dans sa séquence naturelle, ne constitue qu'une entrée-renvoi, avec ou sans début de développement microstructural. Ainsi, plat de côtes, plat à main, parti pris (GRLF) sont accompagnés de leur catégorisation lexico-grammaticale et de leur prononciation. Mais ce ne sont que des entrées-renvois à des articles plus complets (plat et parti) où il sont relocalisables. L'autonomie du syntagme est incertaine. 
LE STATUT DU SYNTAGME DANS LES DICTIONNAIRS GÉNÉRAUX MONOLINGUES

4. Le syntagme est repérable dans sa séquence inversée tout en conservant son statut d'entrée-renvoi. Il peut être suivi ou non d'un début de développement microstructural. Ainsi, l'anthroponyme Pandore sert d'accès au syntagme boîte de Pandore dans le $G R L F$. On trouve l'entrée sous la forme Pandore (boîte de), suivie de la catégorisation locution nominale et de deux renvois, l'un à boîte, l'autre à Pandore. L'autonomie du syntagme est incertaine.

5. Le syntagme et le mot composé sont fusionnés dans une formulation à double lecture. Un développement suit l'entrée (ex. DFP : club(-)sandwich qui s'écrit club sandwich ou club-sandwich). L'autonomie du syntagme est reconnue. L'arrivée du trait d'union concrétise la cohésion des éléments.

6. Le syntagme et le composé sont présentés en double lecture par l'intermédiaire d'un artifice, les parenthèses, qui enferment le trait-d'union. Aucun développement ne suit la séquence. Il s'agit d'une simple entrée qui oriente le consulteur vers un article principal (ex. DFP : tous(-)terrains, tout(-)terrain qui renvoient à terrain). Malgré la présence du signe de liaison des élémentes, le syntagme ne paraît pas autonome.

Tableau 5: Formes de l'entrée syntagmatique dans les DGM

\begin{tabular}{|c|c|c|c|}
\hline Formes & Statut dans l'article & Exemples & Dictionnaires \\
\hline séquence naturelle & $\begin{array}{c}\text { - article complet [1] } \\
\text { - entrée-renvoi [3] } \\
\text { (+ cat. gr. et pron.) } \\
\text { - variante graphique } \\
\text { (+ article complet) } \\
\text { [7] }\end{array}$ & $\begin{array}{l}\text { chemin de fer } \\
\text { parti pris } \rightarrow \text { parti } \\
\text { - compte chèques / } \\
\text { compte-chèques } \\
\text { - big-bang / } \\
\text { big bang }\end{array}$ & $\begin{array}{l}G R L F / P L I / D F P \\
G R L \\
P L I \\
P L I\end{array}$ \\
\hline séquence permutée & $\begin{array}{l}\text { — article complet [2] } \\
\text { — entrée-renvoi } \\
\text { (+ cat. gr.) [4] }\end{array}$ & $\begin{array}{l}\text { - frise (cheval de) } \\
\text { - Bénioff (plan de) } \\
\text { - Bengale (feu de) } \\
\text { Pandore (boîte de) } \rightarrow \\
\text { Pandore et bôte }\end{array}$ & $\begin{array}{l}\text { GRLF } \\
P L I \\
G R L F \\
\text { GRLF }\end{array}$ \\
\hline double lecture & $\begin{array}{l}\text { - article complet [5] } \\
\text { - entrée-renvoi [6] }\end{array}$ & $\begin{array}{l}\text { club (-) sandwich } \\
\text { tous (-) terrains } \rightarrow \\
\text { terrain }\end{array}$ & $\begin{array}{l}D F P \\
D F P\end{array}$ \\
\hline $\begin{array}{l}\text { Réduction } \\
\text { syntagmatique } \\
\text { - base conservée } \\
\text { - expansion } \\
\text { conservée }\end{array}$ & $\begin{array}{l}\text { - article complet [8] } \\
\text { — article complet [8] }\end{array}$ & $\begin{array}{l}\text { repris } \rightarrow \text { repris de } \\
\text { justice } \\
\text { patronesse } \rightarrow \text { dame } \\
\text { patronesse }\end{array}$ & $\begin{array}{l}P L I \\
G R L F / P L I / D F P\end{array}$ \\
\hline
\end{tabular}


7. Le syntagme est présenté suivant l'ordre naturel de la séquence mais il est suivi (ex. PLI : compte-chèques, compte rendu) ou précédé (ex. PLI : big bang). Un développement suit l'entrée concrétisant l'autonomie du syntagme. Le trait d'union vient en outre confirmer la cohésion des parties au plan sémantique.

8. Un mot simple qui n'a pas d'existence autonome en dehors de son insertion dans une formule syntagmatique peut servir de guide pour accéder à l'article. Dans ce cas, la base ou l'expansion qui résultent de la troncation servent à introduire la sousentrée syntagmatique complète qui est elle-même pourvue du discours lexicographique habituel. Ainsi, repris de justice dans le $P L I$ tandis que patronesse donne un article dame patronesse dans le $G R L F$, le $P L I$ et le $D F P$. C'est l'amorce microstructurale qui témoigne ici du statut syntagmatique de la forme ou plutôt du fragment choisi comme entrée. Mais nous sommes déjà ici à cheval sur la microstructure.

\section{CONCLUSION}

On s'est intéressé ici au syntagme en tant qu'unité lexicale codée et que forme composite figée, caractérisée par la présence concomitante d'éléments lexicaux qui offrent une cohésion sémantique suffisante pour que le groupe graphique puisse être identifié à un concept unique. Sans être strictement limitées au volet terminologique, les observations sont quand même demeurées au plan lexical nominal, délaissant pour le moment les syntagmes grammaticaux du genre bien que, en bref, de bric et de broc, $\grave{a}$ brûle-pourpoint.

Dans cette première approche du rôle du syntagme en lexicographie générale, on s'est arrêté sur le seul aspect macrostructural, sur la manifestation la plus immédiatement visible des mots complexes dans les DGM. Quelques vérifications dont on vient de rendre compte démontrent la complexité du phénomène et l'absence de politique cohérente d'un dictionnaire à l'autre.

L'arbre cache ici la forêt. Il reste en effet à pousuivre l'examen amorcé en pénétrant au cœur de l'article pour y repérer les différentes modalités de traitement du syntagme. De brefs coups de sonde promettent des résultats étonnants.

En attendant, il paraît évident que la lexicographie générale est en pleine métamorphose en ce moment. Elle cherche à allier l'innovation et la tradition sous l'influence de plusieurs sources de pression dont la terminologie n'est pas la moindre. Avec comme objectif de mieux décrire le lexique et de rendre le contenu didactique des DGM plus efficace. Chose certaine, le syntagme ne laisse plus les dictionnairistes indifférents et cela est bien pour l'avenir de la (méta) lexicographie.

(1) Je remercie Marie-Claude L'Homme, étudiante à la maîtrise, qui s'est chargée de la compilation des données.

\section{BIBLIOGRAPHIE}

Linguistique

BOULANGER, Jean-Claude: Aspects de l'interdiction dans la lexicographie française contemporaine, coll. «Lexicographica», Séries Maior, $\mathrm{n}^{\circ} 13$, Tübingen, Max Niemeyer.

COLLIGNON, Lucien et GLATINY, Michel (1978): Le dictionnaire, Initiation à la lexicographie, coll. «Textes et non textes», Cédic, Paris.

DUBOIS, Jean et Claude DUBOIS (1971): Introduction à la lexicographie, le dictionnaire, coll. «Langue et langage», Librairie Larousse, Paris.

GUILBERT, Louis (1969): «Dictionnaires et linguistiques: essai de typologie des dictionnaires monolingues français contemporains», Langue française, $\mathrm{n}^{\circ} 2$ [Le lexique]

GUILBERT, Louis (1970) : «La dérivation syntagmatique dans les vocabulaires scientifiques et techniques», Les langues de spécialité (Analyse linguistique et recherche pédagogique). Actes du stage de Saint-Cloud, 23-30 novembre 1967, Publication du Conseil de l'Europe, Strasbourg, Aidela. 
KOKOUREK, Rostislay (1982) : La langue française de la technique et de la science, Oscar Brandstetter, Wiesbaden.

PHAL, André (1969) : «La recherche au CREDIF : la part du lexique commun dans les vocabulaires scientifiques et techniques», Langue française, $\mathrm{n}^{\circ} 2$.

REY, Alain (1985): «Préface de la deuxième édition», Le Grand Robert de la langue française, Dictionnaire alphabétique et analogique de la langue française, $2^{\mathrm{e}}$ éd. entièrement revue et augmentée, Dictionnaires Le Robert, Paris.

REY, Alain (1987): «Le dictionnaire culturel», Lexicographica, $n^{\circ} 3$.

REY, Alain (1988): «Préface», Le Micro-Robert, Langue française plus noms propres, chronologie, cartes, Dictionnaires Le Robert, Paris.

REY-DEBOVE, Josette (1971): Étude linguistique et sémiotique des dictionnaires français contemporains, coll. «Approaches to Semiotics», $\mathrm{n}^{\circ} 13$, The Hague/Paris, Mouton.

Dictionnaires

COTÉ, Normand (1982): Appellations d'emplois dans l'industrie papetière québécoise, coll. «Cahiers de l'Office de la langue française, Éditeur officiel du Québec, Québec.

COTÉ, Normand (1983) : Vocabulaires des papiers et des cartons (anglais-français), coll. «Cahiers de l'Office de la langue française, Gouvernement du Québec, Québec.

Dictionnaire du français plus. À l'usage des francophones d'Amérique (1988) : Édition établie sous la responsabilité de A.E. Shiaty, avec la collaboration de Pierre Auger et de Normand Beauchemin, Rédacteur principal : Claude Poirier, Montréal, Centre éducatif et culturel.

Petit Larousse illustré 1988 (1987) : Larousse, Paris.

Petit Larousse illustré 1989 (1988) : Larousse, Paris.

ROBERT, Paul (1985): Le Grand Robert de la langue française. Dictionnaire alphabétique et analogique de la langue française, $2^{\mathrm{e}}$ éd. entièrement revue et augmentée par $\mathrm{A}$. Rey, Dictionnaires Le Robert, Paris.

ROBERT, Paul (1984) : Le Petit Robert 1. Dictionnaire alphabétique et analogique de la langue française. Nouvelle édition revue, corrigée et mise à jour. Rédaction dirigée par Alain Rey et Josette Rey-Debove, S.N.L. - Dictionnaires Le Robert, Paris.

Vocabulaire des sciences et techniques spatiales, Conseil international de la langue française - La Maison du dictionnaire, Paris. 